\title{
EVIDENCE for LATE FORMATION and YOUNG METAMORPHISM in the ACHONDRITE NAKHLA
}

\author{
D. A. Papanastassiou and G. J. Wassemblik: \\ The Lunatic Asylum of the Charles Arms Laboratory, Division of Geological and Planetary Sciences \\ California Institute of Technology, Pasadena, California 91109
}

(contribution No. 2476)

Abstract. We have determined an age $\mathrm{T}=1.37 \mathrm{AE}$ for Nakhla and an initial ${ }^{87} \mathrm{Sr} /{ }^{86} \mathrm{Sr}, \mathrm{I}=\mathbf{0 . 7 0 2 3}$. A rather thorough Sr equilibration took place at this time between all the phases of this meteorite, but there is clear evidence of somewhat incomplete isotopic equilibrium during the $1.37 \mathrm{AE}$ event, which is not consistent with the derivation of Nakhla from a magma at this time. Nakhla has a young model age of 3.6-2.9 AE which requires that gross differentiation processes for its parent body occur between 3.6 and $1.37 \mathrm{AE}$, significantly later than $4.6 \mathrm{AE}$. The most reasonable model is that Nakhla formed as an igneous rock at $\sim 3 \mathrm{AE}$ and was metamorphosed at $1.37 \mathrm{AE}$. Several similarities exist between the Nakhla parent planet and the Earth and indicate the existence of other objects with close terrestrial chemical affinities.

We report here an age of $1.37 \mathrm{AE}$ for the achondrite Nakhla as determined by the Rb-Sr method. This investigation is part of a systematic search in meteorites for evidence of long term planetary evolution and for indications of the intense bombardment history at $\sim 4.0 \mathrm{AE}$ postulated for the moon by Tera $e t$ al. $[1973,1974 \mathrm{a}, \mathrm{b}]$. The $\mathrm{Rb}-\mathrm{Sr}$ internal isochron age of $3.8 \mathrm{AE}$ on Kodaikanal [Burnett and Wasserburg. 1967] clearly demonstrated the existence of differentiation processes for the parent planets of meteorites on a long time scale. More recently, Papanastassiou et al. [1974] have shown that the achondrite Kapoeta contains basaltic clasts with $\mathrm{Rb}-\mathrm{Sr}$ internal isochron ages of $3.6 \mathrm{AE}$ and $3.9 \mathrm{AE}$. Our interest in Nakhla was stimulated by its peculiar rare earth element pattern [Schmitt and Smith, 1963] and the low ${ }^{40} \mathrm{Ar}-{ }^{39} \mathrm{Ar}$ gas retention age of 1.3 AE for Lafayette and Nakhla [Podosek and Huneke, 1973; Podosek, 1973].

A sample of Nakhla (A) was provided to us by $R$. Schmitt. A preliminary analysis of a total rock (Table 1 ) yielded a model age of TвавI $=3.6 \mathrm{AE}$ and indicated that this meteorite was considerably younger than reported earlier by Pinson et al. [1965]. In order to verify that this discrepancy was not due to heterogeneity of the meteorite, the sample from the Harvard collection (\# 489b) which was used by Pinson et al. [1965] was also analyzed (sample B). From our results, it appears that the earlier analyses are in error.

Study of the meteorite fragments with the binocular microscope showed that a brown stain permeated many of the pale green pyroxene grains along cracks and cleavages. Clusters of white, fine grained, feathery plagioclase crystals, intergrown with an $\mathrm{SiO}_{2}$ polymorph, occasionally surrounded the pyroxene grains as rims and were present in the junctions between pyroxene grains. Inspection of thin sections showed the brown stain to permeate many of the pyroxene and olivine grains on a fine scale. This brown stain proved to be a "glass" with extremely high $\mathrm{K}$ (up to $9 \%$ ). Material interior to the fragments provided to us was chipped for analysis. Mineral separates were prepared using heavy liquids and a Frantz magnetic separator. Electron microprobe analyses were done on the mineral separates by A. Chodos. The compositions of the major phases are shown in Table 2 and are in good agreement with those obtained by Prior [1912]. While the pyroxenes were generally quite uniform, a pyroxene was also found which was

Copyright $\odot 1974$ by the American Geophysical Union richer in $\mathrm{Fe}$ and poorer in $\mathrm{Mg}$, (19.4\% $\mathrm{FeO}$ and $9.7 \% \mathrm{MgO})$. No Ni was detected in the olivine. The thin films of brown glass were difficult to analyze and gave poor sums but were typically $\mathrm{K}$ rich $\left(2-9 \% \quad \mathrm{~K}_{2} \mathrm{O}\right)$. They appeared to be of variable composition and relatively rich in $\mathrm{FeO}$. A typical analysis for one of these brownies is listed in Table 2 . We consider this type of material to be the devitrified glass of a late stage mesostasis. The high $\mathbf{K}$ glass was concentrated in the lower density fractions; however, the bulk of the 2.6 and $2.5 \mathrm{~g} / \mathrm{cm}^{3}$ mineral separates consisted of plagioclase with a tendency to be somewhat more sodic than the typical plagioclases. The glass was intimately intergrown in these plagioclase crystals in small brown blebs and coatings. Considerable care was necessary to remove alkali rich interstitial glass from the pyroxene separates. A high purity pyroxene separate was obtained by successive crushing and density separations followed by magnetic separation and hand picking.

The analytical data for $\mathrm{Rb}$ and $\mathrm{Sr}$ and the $\mathrm{K}$ concentrations are given in Table $\mathbf{l}$. The blank levels were $0.01 \mathrm{ng} \mathrm{Rb}$, $0.1 \mathrm{ng} \mathrm{Sr}$ and $10 \mathrm{ng} \mathrm{K}$, and have a negligible effect on the data. Most samples were first dissolved; a less than one percent aliquot of the total solution was spiked and the $\mathrm{K}, \mathrm{Rb}$ and $\mathrm{Sr}$ concentrations were determined without chemical separation. The remaining solution was then spiked optimally. The glass separate from fragment A was spiked and then split into two aliquots which were processed independently; the results are in good agreement. The first total sample of fragment $A$ was not spiked totally. The analyses of two independently spiked aliquots of the dissolved sample consisting of 8 and 50 percent of the total solution are in good agreement. The other total samples were totally spiked.

Table 1. Rb, Sr, K Results

\begin{tabular}{|c|c|c|c|c|c|c|c|}
\hline \multirow[t]{2}{*}{ Sample } & \multirow{2}{*}{$\begin{array}{c}\text { Wgt. } \\
\text { mg }\end{array}$} & \multirow[t]{2}{*}{$\mathbf{K}^{\mathbf{c}}$} & $\mathbf{R b}^{\mathbf{d}}$ & ${ }^{88} \mathrm{Sr}^{\mathrm{d}}$ & \multicolumn{3}{|c|}{${ }^{87} \mathrm{Rb} /{ }^{86} \mathrm{Sr}{ }^{87} \mathrm{Sr}^{86} \mathrm{Sr}^{\mathrm{e}} \mathrm{T}_{\mathrm{BABI}}$} \\
\hline & & & \multicolumn{2}{|c|}{$10^{-8} \mathrm{~mole} / \mathrm{g}$} & $\times 10^{2}$ & & $\mathbf{A F}$ \\
\hline \multicolumn{8}{|c|}{ Fragment-A } \\
\hline yroxene & 28 & 1 & 0.3067 & 19.16 & .733 & \pm 7 & 8.12 \\
\hline 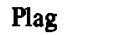 & 2.4 & $1.412 \%$ & 34.44 & 697.6 & & & 3.39 \\
\hline ro & 6 & - & 4 & 42.44 & & & 3.54 \\
\hline Tot & 41 & 911 & 2.254 & 42.50 & 37 & & 3.58 \\
\hline Total-2 & 52 & & 3.7 & 68. & 3 & \pm 5 & 3.39 \\
\hline $\mathbf{a}$ & 0.25 & $\begin{array}{l}4.99 \% \\
4.99 \%\end{array}$ & $\begin{array}{l}149.9 \\
149.8\end{array}$ & & & $\begin{array}{l}8 \pm 11^{1} \\
8 \pm 11^{f}\end{array}$ & 88 \\
\hline \multicolumn{8}{|c|}{ Fragment-B } \\
\hline Pyrox & 20 & & 0.1 & 17 & & & 14.1 \\
\hline lag & 0.4 & $0.980 \%$ & 23.70 & 53 & & & 3.64 \\
\hline Total & 136 & 1477 & 3.799 & 55.68 & 15.91 & & 2.97 \\
\hline Glass- & 1.2 & $2.482 \%$ & 77.90 & 437.5 & 41.53 & 0.7102 & 1.93 \\
\hline Glass- $2^{\text {h }}$ & 0.16 & $0.82 \%$ & 30.08 & 119.2 & 58.86 & $0.71372 \pm 13$ & 1.78 \\
\hline
\end{tabular}

a $8 \%$ aliquot of solution; ${ }^{b} 50 \%$ aliquot; ${ }^{c}$ in ppm unless otherwise noted; $\mathrm{d}_{\text {calculated using }}{ }^{85} \mathrm{Rb} /{ }^{87} \mathrm{Rb}=2.591,{ }^{86} \mathrm{Sr} /{ }^{88} \mathrm{Sr}=0.1194$ and ${ }^{84} \mathrm{Sr} /{ }^{88} \mathrm{Sr}=$ 0.006748 , maximum uncertainties in $\mathrm{Rb}$ and $\mathrm{Sr}$ concentrations are $0.4 \%$ and $0.1 \%$ respectively; ${ }^{\mathrm{e}}$ errors are $2 \sigma_{\text {mean }}$ and correspond to the last significant figure given; $f$ the original solution was spiked and split into halves for further processing; $g$ density $\rho<2.6 \mathrm{~g} / \mathrm{cm}^{3} ; h_{\text {density } \rho<}$ $2.5 \mathrm{~g} / \mathrm{cm}^{3}$. 
Table 2. Chemical Composition (Weight percent)

\begin{tabular}{lclll}
\hline & Brownies & Plag & Pyroxene & Olivine \\
\hline $\mathrm{Na}_{2} \mathrm{O}$ & 2.8 & 7.0 & 0.2 & $\mathrm{ND}$ \\
$\mathrm{K}_{2} \mathrm{O}$ & 6.6 & 0.6 & $\mathrm{ND}$ & $\mathrm{ND}$ \\
$\mathrm{MgO}$ & 0.6 & $<0.1$ & 13.3 & 14.6 \\
$\mathrm{Al}_{2} \mathrm{O}_{3}$ & 12.9 & 24.4 & 0.7 & $<0.1$ \\
$\mathrm{SiO}_{2}$ & 71.6 & 59.6 & 52.2 & 31.9 \\
$\mathrm{CaO}$ & 0.6 & 6.9 & 18.8 & 0.5 \\
$\mathrm{TiO}_{2}$ & 0.3 & $<0.1$ & 0.2 & $<0.1$ \\
$\mathrm{Cr}_{2} \mathrm{O}_{3}$ & $\mathrm{ND}$ & $\mathrm{ND}$ & 0.3 & 0.1 \\
$\mathrm{MnO}$ & $\mathrm{ND}$ & $\mathrm{ND}$ & 0.3 & 0.9 \\
$\mathrm{FeO}$ & 2.0 & 0.7 & 14.3 & 51.9 \\
$\mathrm{BaO}$ & 0.1 & 0.3 & $\mathrm{ND}$ & $\mathrm{ND}$ \\
\hline
\end{tabular}

ND: Not Determined

The total meteorite ${ }^{87} \mathrm{Sr} /{ }^{86} \mathrm{Sr}$ is relatively radiogenic so that the calculation of the model age is not sensitive to the choice of BAl3I as the initial ${ }^{87} \mathrm{Sr} /{ }^{86} \mathrm{Sr}$, as compared to the more primitive value ALL obtained for Allende [Gray et al., 1973]. The model ages for all the samples are given in Table 1 . The variability in the model age for the small single fragments analyzed for the total meteorite is due to sampling bias due to variable amounts of the high $\mathrm{K}$ phase. The total meteorite model ages are distinctly lower than $4.6 \mathrm{AE}$ and range from $\mathrm{T}_{\mathrm{BABI}}=3.58 \mathrm{AE}$ to TraisI $=2.97 \mathrm{AE}$. These ages require that the meteorite have been produced by differentiation of some parent planetary body at times strictly less than $3.58 \mathrm{AE}$ and possibly less than 2.97 AE. Therefore, Nakhla may not be considered as a total melt of a 4.6 AE "relict." Insofar as the model age of Nakhla is uncertain due to possibly nonrepresentative sampling, the conclusions based on this model age are not strictly correct. However, it is unlikely that all our total samples are significantly biased towards the high $\mathrm{K}$ phase, and therefore, we shall assume for this work that the young model age of Nakhla reflects a late differentiation of its parent. The lowest model age for mineral phases in Nakhla is 1.78 AE and is obtained for a separate rich in high $\mathrm{K}$ glass. This is a strict upper limit to the time at which Nakhla was last disturbed, so that Nakhla must be a young meteorite.

All the data shown in fig. 1 define a good linear array with a slope corresponding to an age of $1.34 \mathrm{AE}$. The initial ${ }^{87} \mathrm{Sr} /{ }^{86} \mathrm{Sr}$ is quite high with regard to BABI and is equal to about 0.7025 . $\mathrm{K}$ Ar ages have been reported for Nakhla using the ${ }^{39} \mathrm{Ar}-{ }^{411} \mathrm{Ar}$ technique [Podosek, 1973]. The lower temperature (less than $900^{\circ} \mathrm{C}$ ) Ar release fractions determine a good plateau corresponding to an age of $1.3 \mathrm{AE}$. Following the petrographic description of the meteorite given above, it is most reasonable to attribute the lower temperature ${ }^{40} \mathrm{Ar}-{ }^{-39} \mathrm{Ar}$ pattern to the high $\mathrm{K}$ glass and the sodic plagioclase. The higher temperature release fractions for Nakhla show a complex pattern dipping to apparent ages of $0.4 \mathrm{AE}$ which is not understood, but may reflect peculiarities attributable to pyroxene [Huneke et al., 1972]. A sample of Lafayette [Podosek and Huneke 1973; Podosek, 1973] yields an age of $1.33 \pm 0.03 \mathrm{AE}$. These results enhance the possibility that the two Nakhlites (which may be only one meteorite) have had similar histories. The agreement of the $\mathrm{Rb}-\mathrm{Sr}$ and $\mathrm{K}-\mathrm{Ar}$ ages provides strong evidence that Nakhla was isotopically and chemically reequilibrated and degassed at $\sim 1.34 \mathrm{AE}$.

Closer inspection of the data indicates that the meteorite is a more complex system since all the data do not fall precisely on a straight line on the $\mathrm{Rb}-\mathrm{Sr}$ evolution diagram. In particular, the plagioclase separates from both $A$ and $B$ fragments lie distinctly off any line drawn through most other data. In lieu of a single precise isochron, we have drawn two reference lines in fig. 1 corresponding to tie lines between the pyroxene and glass for both fragments $A$ and $B$ respectively. These lines are parallel but correspond to different intercepts on the $y$ axis. From the slope, we calculate a time $\mathrm{T}^{\circ}=1.31 \mathrm{AE}$ and a difference in ${ }^{87} \mathrm{Sr} /{ }^{86} \mathrm{Sr}$ intercepts of 0.00012 . The relative deviations $(\theta)$ in ${ }^{87} \mathrm{Sr} /{ }^{86} \mathrm{Sr}$ of the data from reference line $\mathrm{B}$ are shown in the insert. The reference lines in the insert appear as parallel horizontal lines and we have placed $\theta=0$ for the fragment $B$ line. For this discussion, we consider the deviations $\theta$ as a useful parameter in describing the dispersion in the data from line $B$. We note in the insert that a single line can be drawn through the pyroxene and glass separates for both fragments and the total rock-B analysis. This line would narrowly miss the total rock-A analysis. However, the plagioclase $A$ and $B$ samples lie far off any line which can be drawn through all the other data. From a geometrical point of view, it is the latter two samples that offer the strongest evidence for lack of complete isotopic homogenization of initial ${ }^{87} \mathrm{Sr} /{ }^{86} \mathrm{Sr}$ in Nakhla. In principle, deviations from an isochron can occur either because the ${ }^{87} \mathrm{Sr} /{ }^{86} \mathrm{Sr}$ was not homogenized at the time of the last disturbance or because $\mathrm{Rb}$ and $\mathrm{Sr}$ have subsequently migrated between different phases. For example, if the deviations from a single isochron were attributed to modern day $\mathbf{R b}$ migration, the plagioclase data would correspond to about $15 \%$ increase in their $\mathrm{Rb}$ content. Since the $\mathrm{K}$-Ar age and the time $\mathrm{T}^{\circ}$ are essentially coincident, we will assume in this discussion that the deviations are due to lack of complete initial isotopic homogenization and not a modern disturbance.

The evidence for lack of initial isotopic homogeneity clearly does not permit the interpretation of the $1.34 \mathrm{AE}$ age as a time of crystallization from a melt. From a petrographic point of view, it is more likely that the plagioclase and the glass were at equilibrium rather than the glass and the pyroxene. Therefore, the plagioclase-glass tie line must be a better approximation to the time of reequilibration of the glass. This line (EF) has been shown for clarity in the insert only. All plagioclase and glass analyses, including the most radiogenic low density fraction, lie on this line, which yields $\mathrm{T}=1.37 \pm 0.02 \mathrm{AE}$ and $I=0.70232 \mp 6[2 \sigma$ errors $]$. Since the plagioclase and glass separates are mixtures of plagioclase and the high $\mathrm{K}$ phase in the rock, the true high $\mathbf{K}$ glass end member must lie above the pyroxene-glass tie lines. Therefore, the total rock analysis in fig. 1 may lie within the triangle formed by the pyroxene, plagioclase and "pure glass" components. The most serious problem with interpreting the plagioclase and the high $\mathrm{K}$ phases as having reached equilibrium at $1.37 \mathrm{AE}$ concerns the pyroxene, which then at $1.37 \mathrm{AE}$ must have had a ${ }^{87} \mathrm{Sr} /{ }^{86} \mathrm{Sr}$ composition higher than the ${ }^{87} \mathrm{Sr} /{ }^{86} \mathrm{Sr}$ composition of the coex-

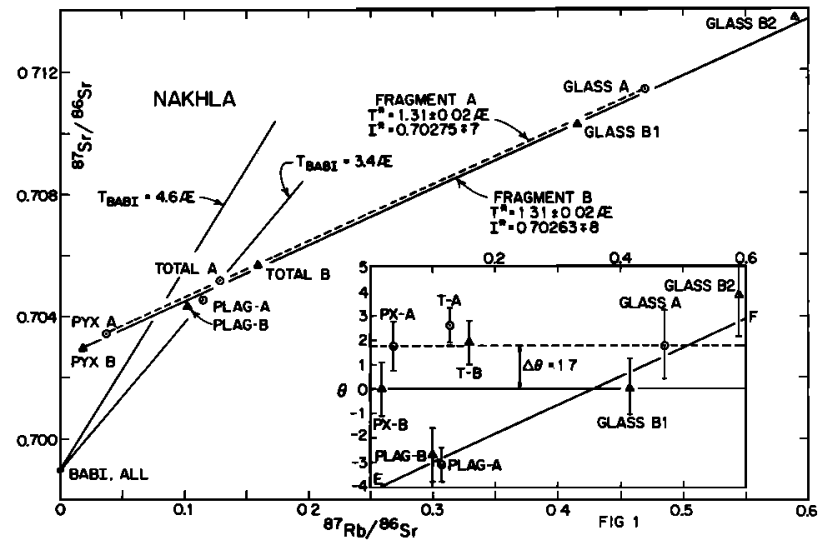

Fig. 1. Rb-Sr evolution diagram for Nakhla. Pyroxeneglass tie lines are shown. Note that the data form a good linear array although they do not lie on a single isochron. The insert shows deviations in ${ }^{87} \mathrm{Sr} /{ }^{86} \mathrm{Sr}\left(\theta\right.$ in parts in $\left.10^{4}\right)$ from the fragment $B$ tie line. Line EF in the insert fits all the plagioclase and glass data and corresponds to an age $1.37 \mathrm{AE}$. It reflects our best estimate of the time of metamorphism of Nakhla. 
isting glass and plagioclase. Insofar as the pyroxene contains $\mathrm{Sr}$ but only very small amounts of $K$ and $R b$, it is unlikely that, if the pyroxene existed prior to the $1.37 \mathrm{AE}$ event, it would have had a sufficiently high $\mathrm{Rb} / \mathrm{Sr}$ to produce a higher ${ }^{87} \mathrm{Sr} /{ }^{\mathrm{st}} \mathrm{Sr}$ than the average of the sodic plagioclase and high $K$ phases. However, because of the small amount of $\mathrm{Sr}$ in the pyroxene $(\sim 20 \mathrm{ppm})$, the addition of only a small amount of radiogenic ${ }^{87} \mathrm{Sr}$ could cause a significant shift in ${ }^{87} \mathrm{Sr} /{ }^{86} \mathrm{Sr}$ in this phase as compared with the plagioclase or glass which have over a factor of $\mathbf{3 0}$ higher $\mathrm{Sr}$ concentrations.

If we had obtained a perfect internal isochron, we would have erroneously interpreted this as resulting from an igneous event. The disequilibrium appears to require some sort of metamorphic process. The simplest model which we can propose is that the immediate parent of Nakhla was an older ig. neous rock with essentially the identical texture and character of the present rock and was formed some time after $\sim 3.4 \mathrm{AE}$. The bulk of the material consisted of the diopside and olivine crystals which were of cumulate origin. During the crystallization process which formed Nakhla, plagioclase crystallized out together with the late pyroxenes followed by the quenching of the last phase which was an inviscid alkali rich glass which penetrated the cleavage planes and imperfections of both the pyroxene and plagioclase crystals. Subsequent to this original igneous process, the meteorite was subject to dry, relatively low temperature, metamorphism at $1.37 \mathrm{AE}$ without gross recrystallization of the pyroxenes, although some incipient melting may have taken place to cause the remobilization of the glass.

The "intimate" association of the glass with the pyroxenes permitted the pyroxenes at $1.37 \mathrm{AE}$ to exchange on a very local scale with a medium rich in ${ }^{87} \mathrm{Sr}$. If both the plagioclase and the pyroxene were close to $\mathrm{BABI}$ prior to $1.37 \mathrm{AE}$, then raising the ${ }^{87} \mathrm{Sr} /{ }^{86} \mathrm{Sr}$ of the pyroxene by exchange with the high $\mathrm{K}$ phases requires about 30 times less radiogenic $\mathrm{Sr}$ per gram than to raise the plagioclase ${ }^{87} \mathrm{Sr} /{ }^{86} \mathrm{Sr}$ by the same amount. The sensitivity of a Sr poor phase like the pyroxene to addition of radiogenic ${ }^{87} \mathrm{Sr}$ may be more than enough to compensate for the fact that the pyroxene is further below the equilibrium ${ }^{87} \mathrm{Sr} /{ }^{86} \mathrm{Sr}$ than the plagioclase just prior to the metamorphic event. The net effect of the micro redistribution was to achieve a state close to complete bulk average equilibration of $\mathrm{Sr}$. The mechanism presented above for obtaining pyroxene crystals at $1.37 \mathrm{AE}$ with a higher initial ${ }^{87} \mathrm{Sr} /{ }^{86} \mathrm{Sr}$ than the equilibrium value requires the existence, just prior to the $1.37 \mathrm{AE}$ event, of phases in the meteorite with significantly different ${ }^{87} \mathrm{Sr} /{ }^{86} \mathrm{Sr}$. This indicates that the (igneous) differentiation which produced the total meteorite must have occurred closer to 3.0 or $3.6 \mathrm{AE}$ rather

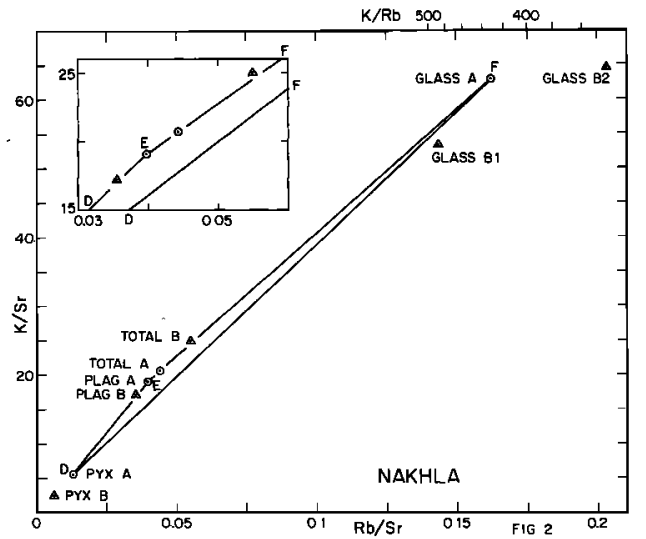

Fig. 2. $\mathrm{Rb} / \mathrm{Sr}-\mathrm{K} / \mathrm{Sr}$ correlation plot. The separates of fragment A (open circles) form a triangle (DEF) indicating the presence of at least three components in Nakhla. The upper right hand corner nomogram yields the $K / R b$ ratio if a straight line is drawn through a data point and the origin.
Rb-Sr AGES OF LUNAR ROCKS AND METEORITES

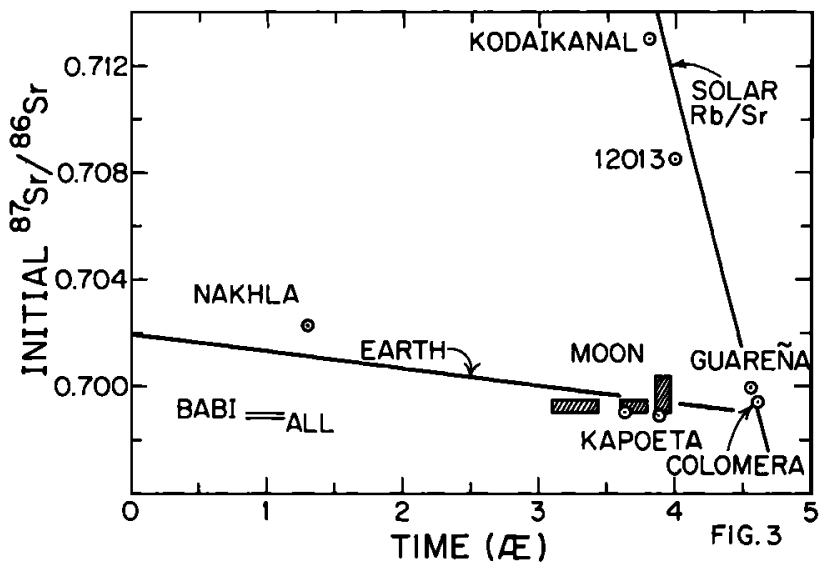

Fig. 3. Time-Initial ${ }^{87} \mathrm{Sr} /{ }^{86} \mathrm{Sr}$ anticorrelation plot. Nakhla yields the youngest meteorite age and a relatively high I value as compared to eucrites, howardites and the moon. By comparison with the evolution of the solar nebula, the parent planet of Nakhla was significantly enriched in $\mathrm{Sr}$, and followed an evolution similar to the earth.

than near $1.37 \mathrm{AE}$. The metamorphism, representing in situ reequilibration, is the process which we believe is being dated by the Rb-Sr and K-Ar methods.

We now examine whether the linear array in fig. 1 can represent a two component mixing line, ignoring the minor isotopic disequilibrium. In the correlation plot of $\mathrm{K} / \mathrm{Sr}$ and $\mathrm{Rb} / \mathrm{Sr}$ (fig. 2), mixtures of only two components will lie on a line segment with the two pure components as end members. The data for fragment $A$ form a triangle (DEF) requiring that at least three components be present in the meteorite. The pyroxene lies below line EF and is displaced towards the origin indicating the presence of a low $\mathrm{Rb}$, low $\mathrm{K}$ but significant $\mathrm{Sr}$ component. Since the total A sample lies on line EF (see insert) a fourth phase, above line EF must be present in the meteorite. Similar conclusions are reached for fragment $\mathrm{B}$, for which the total- $B$ analysis lies distinctly above the plagioclase-glass mixing line. We also note that the pyroxene contains a significant amount of $\mathrm{Sr}(\sim 20 \mathrm{ppm})$ and this amount of $\mathrm{Sr}$ contributes about $25 \%$ of the total $\mathrm{Sr}$ in the meteorite. We conclude that the intrinsic linear nature of the data arrays on the $\mathrm{Rb}-\mathrm{Sr}$ evolution diagram (fig. 1) is not the result of mixing of only two phases.

We note that $\mathrm{Rb} / \mathrm{Sr}$ of the parent material of Nakhla is less than 0.02 and similar to values deduced for the earth's upper mantle. The $\mathrm{Rb}-\mathrm{Sr}$ evolution diagram in fig. 3 shows a comparison of Nakhla with other meteorites, lunar samples and the terrestrial and solar growth curves. The U content of Nakhla is $49 \mathrm{ppb}$ [Morgan and Lovering, 1964; Clark et al. 1967] which yields $\mathrm{K} / \mathrm{U}=2-3 \times 10^{4}$. This ratio is distinct from either chondritic $\left(8 \times 10^{4}\right)$ or lunar $\left(2 \times 10^{3}\right)$ values, but similar to terrestrial $\mathrm{K} / \mathrm{U} \sim 10^{4}$ [Wasserburg et al., 1964]. We also note that the rare earth element pattern of Nakhla [Schmitt and Smith, 1963] is identical to Hawaiian basalts and does not show a $\mathrm{Eu}$ anomaly. We conclude that marked analogies exist between the earth and the parent planet of Nakhla.

The low K-Ar age of Nakhla has been known for some time [Stauffer, 1962]. Ganapathy and Anders [1969] have interpreted this result as degassing due to a major impact on the parent body. Podosek [1973] in discussing the ${ }^{40} \mathrm{Ar}-{ }^{39} \mathrm{Ar}$ ages of Lafayette and Nakhla has pointed out that a major event occurred for both meteorites which resulted in complete or nearly complete degassing of $\mathrm{Ar}$ at $1.3 \mathrm{AE}$. This age is essentially the same as the total ${ }^{40} \mathrm{Ar}-{ }^{40} \mathrm{~K}$ age of $1.5(+0.1,-0.3) \mathrm{AE}$ by Stauffer [1962]. Podosek [1973] explored two extreme models 
ranging from thermal degassing to the formation of these meteorites from a melt. The present data confirm the ${ }^{40} \mathrm{Ar}-{ }^{39} \mathrm{Ar}$ age and prove that rather thorough $\mathrm{Sr}$ isotopic equilibration took place at $1.37 \mathrm{AE}$ which required an intense metamorphism, possibly accompanied by partial melting. In addition, the $\mathrm{Rb}-\mathrm{Sr}$ data demonstrate that Nakhla was formed by a major planetary differentiation process (impact or internal magmatism) more than $1 \mathrm{AE}$ after the original formation of the solar system. While low gas retention ages of many meteorites may be interpreted as the result of degassing during minor thermal events, distinctive $\mathrm{Rb}-\mathrm{Sr}$ total rock model ages must represent extensive physical and chemical processes on parent planets at times different from $\sim 4.6 \mathrm{AE}$.

\section{Conclusions}

1) The internal isochron age of $1.37 \mathrm{AE}$ for Nakhla represents an episode of metamorphic activity which may have been produced by either impact heating or by internal heat sources on the parent body.

2) Based on the total rock model age, the parent body of Nakhla was subject to physical and chemical differentiation more recently than $3.6 \mathrm{AE}$. Nakhla was not produced by closed system re-equilibration of a $4.6 \mathrm{AE}$ source material.

3) The parent object of Nakhla could not have had the bulk chemical composition of eucrites, howardites or chondrites based on initial $\mathrm{Sr}$ and rare earth elements. The moon does not appear to be a possible parent body.

4) The parent body for Nakhla has some chemical characteristics which are similar to the earth $(\mathrm{Rb} / \mathrm{Sr}, \mathrm{K} / \mathrm{U}$, $\mathrm{REE}$ ) and indicates that there may be other planetary objects in the solar system with close to terrestrial composition.

5) It may no longer be assumed that the major physical and chemical differentiation and equilibration processes manifested in meteorites took place only early in solar system history.

6) The evidence for subtle but definite element redistribution and the implied metamorphism of the objects in the relatively recent past must be seriously taken into account in discussing the origin of meteorites [cf. Gray et al., 1973].

7) The young age of $1.37 \mathrm{AE}$ is far from the time of the terminal lunar cataclysm and the evidence of this intense bombardment on a solar system scale is only indicated by Kodaikanal and possibly Kapoeta.

Acknowledgments. The sample from the Harvard collection was provided through the generous courtesy of Professor C. Frondel with prompting from Judith Frondel. We thank R. A. Schmitt for stimulating our interest in Nakhla in a Mexican restaurant and for providing us with a sample. A. L. Albee provided incisive comments at a critical stage of this paper. This work was supported by the National Science Foundation, Grant GP 28027.

\section{References}

Burnett, D. S., and G. J. Wasserburg, Evidence for the formation of an iron meteorite at $3.8 \times 10^{9}$ years, Earth Planet. Sci. Letters, 2, 137-147, 1967.
Clark, R. S., M. W. Rowe, R. Ganapathy and P. K. Kuroda, Iodine, uranium and tellurium contents of meteorites, Geochim. Cosmochim. Acta, 31, 1605-1613, 1967.

Ganapathy, R. and E. Anders, Ages of calcium-rich achondrites-II. Howardites, Nakhlites and the Angra dos Reis angrite, Geochim. Cosmochim. Acta, 33, 775-787, 1969.

Gray, C. M., D. A. Papanastassiou and G. J. Wasserburg, The identification of early condensates from the solar nebula, Icarus, 20, 213-239, 1973.

Huneke, J. C., F. A. Podosek, G. Turner and G. J. Wasserburg, ${ }^{40} \mathrm{Ar}-{ }^{39} \mathrm{Ar}$ systematics in lunar rocks and separated minerals of lunar rocks from Apollo 14 and 15, Lunar Science III, pp. 413-415, C. Watkins ed., Lunar Science Institute, Houston, 1972.

Morgan, J. W. and J. F. Lovering, Uranium and thorium abundances in stony meteorites 2 . The achondritic meteorites, $J$. Geophys. Res., 69, 1989-1994, 1964.

Papanastassiou, D. A., R. S. Rajan, J. C. Huneke and G. J. Wasserburg, $\mathrm{Rb}-\mathrm{Sr}$ ages and lunar analogs in a basaltic achondrite; implications for early solar system chronologies, Lunar Science V, pp. 583-585, Lunar Science Institute, Houston, 1974.

Pinson, W. H., Jr., C. C. Schnetzler, E. Beiser, H. W. Fairbairn and P. M. Hurley, Rb-Sr age of stony meteorites, Geochim. Cosmochim. Acta, 29, 455-466, 1965.

Podosek, F. A., Thermal history of the nakhlites by the ${ }^{40} \mathrm{Ar}-$ ${ }^{39}$ Ar method, Earth Planet. Sci. Letters, 19, 135-144, 1973.

Podosek, F. A. and J. C. Huneke, ${ }^{40} \mathrm{Ar}-{ }^{39} \mathrm{Ar}$ chronology of four calcium-rich achondrites, Geochim. Cosmochim. Acta, 37, 667-684, 1973.

Prior, G. T., The meteoric stones of El Nakhla el Baharia (Egypt), Min. Mag., 16, 274, 1912.

Schmitt, R. A. and R. H. Smith, Implications of similarity in rare earth fractionation of nakhlitic meteorites and terrestrial basalts, Nature, 199, 550-552, 1963.

Stauffer, H., On the production ratios of rare gas isotopes in stone meteorites, J. Geophys. Res., 67, 2023-2028, 1962.

Tera, F., D. A. Papanastassiou and G. J. Wasserburg, A lunar cataclysm at $\sim 3.95 \mathrm{AE}$ and the structure of the lunar crust, Lunar Science IV, pp. 723-725, J. W. Chamberlain ed., Lunar Science Institute, Houston, 1973.

Tera, F., D. A. Papanastassiou and G. J. Wasserburg, The lunar time scale and a summary of isotopic evidence for a terminal lunar cataclysm, Lunar Science V, pp. 792-794, Lunar Science Institute, Houston, 1974a.

Tera, F., D. A. Papanastassiou and G. J. Wasserburg, Isotopic evidence for a terminal lunar cataclysm, Earth Planet. Sci. Letters, 22, 1-21, 1974b.

Wasserburg, G. J., G. J. F. MacDonald, F. Hoyle and W. A. Fowler, Relative contributions of uranium, thorium and potassium to heat production in the earth, Science, 143, 465-467, 1964.

(Received April 15, 1974; accepted April 24, 1974.) 